\title{
On a soft mapping and its fixed points
}

\section{Dariusz Wardowski*}

\section{"Correspondence:}

wardd@math.uni.lodz.pl

Department of Nonlinear Analysis,

Faculty of Mathematics and

Computer Science, University of

Łódź, Banacha 22, Łódź, 90-238,

Poland

\begin{abstract}
In the article first we introduce a new notion of soft element of a soft set and establish its natural relation with soft operations and soft objects in soft topological spaces. Next, using the notion of soft element, we define, in a different way than in the literature, a soft mapping transforming a soft set into a soft set and provide basic properties of such mappings. The new approach to soft mappings enables us to obtain the natural first fixed-point results in the soft set theory. Throughout the paper a comprehensive set of examples illustrating the discussed topics is presented.

MSC: $47 \mathrm{H} 10 ; 54 \mathrm{A05}$
\end{abstract}

Keywords: soft set; soft element of soft set; soft mapping; fixed point of soft mapping; soft compact topological space

\section{Introduction}

The soft set theory, initiated by Molodtsov [1] in 1999, is one of the branches of mathematics, which aims to describe phenomena and concepts of an ambiguous, undefined, vague and imprecise meaning. This theory is applicable where there is no clearly defined mathematical model. Recently, many papers concerning soft sets have been published; see, e.g., [2-6]. Interesting papers merging soft set theory with fuzzy sets have also appeared; see, e.g., [7-9]. In [3] the authors introduced the soft set relations and many related concepts. The interesting paper is also [4], where the authors introduced the notion of soft topology on a soft set and proved basic properties concerning soft topological spaces. In many aspects of mathematics, fixed point theory has a tremendous application. Hence, by the present paper, we want to initiate the investigations concerning a fixed point in soft set theory for a certain class of new defined soft mappings in soft topological spaces. The author believes that the presented new results can be the inspiration for many authors.

In this article, firstly, we establish the concept of soft element of a soft set and show its natural connection with the notion of soft set and soft operations. Next, we investigate soft topological spaces and formulate the definitions and deliver some properties of a soft compact topological space. Further, on the basis of the introduced notion of soft element, we give a natural definition of a soft mapping, the image and the inverse of a soft mapping and discuss its properties. In the last, main section of this work, we define the notion of fixed point of a soft mapping and, inspired by [10], prove a new fixed-point result for a soft mapping in a soft compact Hausdorff topological space. The paper includes many examples illustrating presented concepts and showing the necessity of some assumptions.

C 2013 Wardowski; licensee Springer. This is an Open Access article distributed under the terms of the Creative Commons Attribution License (http://creativecommons.org/licenses/by/2.0), which permits unrestricted use, distribution, and reproduction in any medium, provided the original work is properly cited. 


\section{Preliminaries}

Throughout the paper let $\mathbb{R}$ be a set of all real numbers, let $\mathbb{Z}$ be a set of all integers and let $\mathbb{N}$ be a set of all natural numbers. Denote by $U$ an initial universe, by $E$ a set of parameters and by $2^{U}$ the collection of all subsets of $U$.

Definition 2.1 Let $A$ be a nonempty subset of $E$. A soft set $F_{A}$ on $U$ is a set of the form

$$
F_{A}=\left\{\left(p, \Lambda_{F}(p)\right): p \in E\right\},
$$

where $\Lambda_{F}: E \rightarrow 2^{U}$ is a set-valued map such that $\Lambda_{F}(p)=\varnothing$ for $p \notin A . \Lambda_{F}$ is called an approximate function of $F_{A}$. The elements of $F_{A}$ of the form $(p, \varnothing)$ will be omitted. Very often if a set of parameters $A$ is of no importance, we will write $F$ instead of $F_{A}$. The collection of all soft sets on $U$ will be denoted by $S(U)$.

Example 2.1 Let $U=\mathbb{N}, E=\{$ small, big, very big $\}, A=$ \{small, very big $\}$. We define on $U$ a soft set $F_{A}$ as follows:

$$
F_{A}=\left\{(\text { small, }\{n \in U: n<100\}),\left(\text { very big, }\left\{n \in U: n>10^{6}\right\}\right)\right\} .
$$

Obviously, an element (big, $\varnothing$ ) is also the element of $F_{A}$.

Definition 2.2 An empty soft set, denoted by $\tilde{\varnothing}$, is a soft set of the form $\{(p, \varnothing): p \in E\}$.

Example 2.2 Let $U=\{$ white, yellow, red, azure $\}, E=\{$ dark, ugly $\}$. In this case the soft empty set is of the form $\tilde{\varnothing}=\{($ dark, $\varnothing)$, (ugly, $\varnothing)\}$.

Definition 2.3 A soft set $F_{A}$ is called the $A$-universal soft set and is denoted by $I_{\tilde{A}}$ if $\Lambda_{F}(p)=U$ for each $p \in A$, i.e., $I_{\tilde{A}}=\{(p, U): p \in A\}$.

Example 2.3 Let $U=\{2 n: n \in \mathbb{N}\}, E=\{$ positive, even $\}$. Then

$$
F_{E}=\{(\text { positive }, U),(\text { even }, U)\}=I_{\tilde{E}} \text {. }
$$

Definition 2.4 Let $F_{1}, F_{2} \in S(U) . F_{1}$ is called a soft subset of $F_{2}$, which is denoted by $F_{1} \tilde{\subseteq}$ $F_{2}$, if $\Lambda_{F_{1}}(p) \subseteq \Lambda_{F_{2}}(p)$ for each $p \in E$.

Obviously, for each $F \in S(U), \tilde{\varnothing} \tilde{\subseteq} F$ and $F \tilde{\subseteq} I_{\tilde{E}}$.

Example 2.4 Let $U=\left\{u_{1}, u_{2}\right\}, E=\left\{e_{1}, e_{2}\right\}$ and let $F_{1}, F_{2} \in S(U)$ be of the form $F_{1}=$ $\left\{\left(e_{1},\left\{u_{1}, u_{2}\right\}\right),\left(e_{2},\left\{u_{1}\right\}\right)\right\}, F_{2}=\left\{\left(e_{1},\left\{u_{1}\right\}\right)\right\}$. Then $F_{2} \tilde{\subseteq} F_{1}$.

Definition 2.5 Let $F_{1}, F_{2} \in S(U)$. We say that the soft sets $F_{1}, F_{2}$ are equal, which is denoted by $F_{1}=F_{2}$, if $\Lambda_{F_{1}}(p)=\Lambda_{F_{2}}(p)$ for all $p \in E$.

It is clear that $F_{1}=F_{2}$ if and only if $F_{1} \tilde{\subseteq} F_{2}$ and $F_{2} \tilde{\subseteq} F_{1}$.

The basic soft operations on the soft sets are defined as follows. 
Definition 2.6 Let $F_{1}, F_{2} \in S(U)$. We define a soft union $\tilde{\cup}$, a soft product $\tilde{\cap}$ and a soft difference $\tilde{\Upsilon}$ of the soft sets $F_{1}, F_{2}$ as follows:

1. $F_{1} \tilde{\cup} F_{2}=\left\{\left(p, \Lambda_{F_{1}}(p) \cup \Lambda_{F_{2}}(p)\right): p \in E\right\}$,

2. $F_{1} \tilde{\cap} F_{2}=\left\{\left(p, \Lambda_{F_{1}}(p) \cap \Lambda_{F_{2}}(p)\right): p \in E\right\}$,

3. $F_{1} \widetilde{\backslash} F_{2}=\left\{\left(p, \Lambda_{F_{1}}(p) \backslash \Lambda_{F_{2}}(p)\right): p \in E\right\}$.

Example 2.5 Let $U=\left\{u_{1}, u_{2}, u_{3}, u_{4}\right\}$ and $E=\left\{p_{1}, p_{2}, p_{3}\right\}$. For the soft sets of the form $F_{1}=$ $\left\{\left(p_{1},\left\{u_{1}, u_{2}\right\}\right),\left(p_{2},\left\{u_{2}, u_{3}\right\}\right)\right\}, F_{2}=\left\{\left(p_{1},\left\{u_{1}, u_{4}\right\}\right),\left(p_{3},\left\{u_{3}, u_{4}\right\}\right)\right\}$ we have

$$
\begin{aligned}
& F_{1} \tilde{\cup} F_{2}=\left\{\left(p_{1},\left\{u_{1}, u_{2}, u_{4}\right\}\right),\left(p_{2},\left\{u_{2}, u_{3}\right\}\right),\left(p_{3},\left\{u_{3}, u_{4}\right\}\right)\right\}, \\
& F_{1} \tilde{\cap} F_{2}=\left\{\left(p_{1},\left\{u_{1}\right\}\right)\right\}, \\
& F_{1} \tilde{\Upsilon} F_{2}=\left\{\left(p_{1},\left\{u_{2}\right\}\right),\left(p_{2},\left\{u_{2}, u_{3}\right\}\right)\right\} .
\end{aligned}
$$

The basic properties of the soft operations are described, e.g., in [4].

Definition 2.7 Let $\left\{F_{i}\right\}_{i \in I} \subseteq S(U)$. A generalized soft union $\tilde{U}$ and a generalized soft product $\tilde{\bigcap}$ of the family of the soft sets $\left\{F_{i}\right\}_{i \in I}$ are defined as follows:

1. $\tilde{\bigcup}_{i \in I} F_{i}=\left\{\left(p, \bigcup_{i \in I} \Lambda_{F_{i}}(p)\right): p \in E\right\}$,

2. $\tilde{\bigcap}_{i \in I} F_{i}=\left\{\left(p, \bigcap_{i \in I} \Lambda_{F_{i}}(p)\right): p \in E\right\}$.

Definition 2.8 Let $F \in S(U)$. A soft complement of $F$, denoted by $F^{\tilde{c}}$, is a soft set of the form

$$
F^{\tilde{c}}=\left\{\left(p, U \backslash \Lambda_{F}(p)\right): p \in E\right\} .
$$

Clearly, the following equalities hold: $F^{\tilde{c}}=I_{\tilde{E}} \tilde{\backslash} F,\left(F^{\tilde{c}}\right)^{\tilde{c}}=F, \tilde{\varnothing}^{\tilde{c}}=I_{\tilde{E}}$.

\section{Soft elements of soft sets}

In this section we establish the definition of a soft element of a soft set and present its natural connection with soft sets and soft operations.

Definition 3.1 Let $F \in S(U)$. We say that $\alpha=(p,\{u\})$ is a nonempty soft element of $F$ if $p \in E$ and $u \in \Lambda_{F}(p)$. The pair $(p, \varnothing)$, where $p \in E$, will be called an empty soft element of $F$. Nonempty soft elements of $F$ and empty soft elements of $F$ will be called the soft elements of $F$. The fact that $\alpha$ is a soft element of $F$ will be denoted by $\alpha \tilde{\in} F$.

The following proposition presents the basic properties of the soft elements.

Proposition 3.1 Let $F, F_{1}, F_{2} \in S(U)$. The following hold:
1. $\forall_{p \in E}(p, \varnothing) \tilde{\in} F$,
2. $\alpha \tilde{\in} F \Leftrightarrow\{\alpha\} \tilde{\subseteq} F$,
3. $\alpha \tilde{\in} F_{1} \tilde{\cup} F_{2} \Leftrightarrow \alpha \tilde{\in} F_{1} \vee \alpha \tilde{\in} F_{2}$,
4. $\alpha \tilde{\in} F_{1} \tilde{\cap} F_{2} \Leftrightarrow \alpha \tilde{\in} F_{1} \wedge \alpha \tilde{\in} F_{2}$,
5. $\alpha \tilde{\epsilon} F_{1} \tilde{\backslash} F_{2} \Leftrightarrow \alpha \tilde{\epsilon} F_{1} \wedge \alpha \tilde{\notin} F_{2}$ for each nonempty soft element $\alpha$. 
Example 3.1 Let $U=\left\{u_{1}, u_{2}, u_{3}\right\}, E=\left\{p_{1}, p_{2}\right\}$. Take a soft set $F \in S(U)$ of the form $F=$ $\left\{\left(p_{1},\left\{u_{1}, u_{2}\right\}\right)\right\}$. Then all the soft elements of $F$ are the following: $\left(p_{1}, \varnothing\right),\left(p_{1},\left\{u_{1}\right\}\right),\left(p_{1},\left\{u_{2}\right\}\right)$, $\left(p_{2}, \varnothing\right)$.

Observe that $F=\left\{\left(p_{1}, \varnothing\right)\right\} \tilde{\cup}\left\{\left(p_{1},\left\{u_{1}\right\}\right)\right\} \tilde{\cup}\left\{\left(p_{1},\left\{u_{2}\right\}\right)\right\} \tilde{\cup}\left\{\left(p_{2}, \varnothing\right)\right\}$.

Proposition 3.2 For each $F \in S(U)$, the following holds:

$$
F=\tilde{\bigcup}\{\{\alpha\}: \alpha \tilde{\in} F\} .
$$

Proof For $F \in S(U)$, we have the following:

$$
\begin{aligned}
F & =\left\{\left(p, \Lambda_{F}(p)\right): p \in E\right\}=\left\{\left(p, \bigcup_{u \in \Lambda_{F}(p)}\{u\}\right): p \in E\right\} \\
& =\tilde{\bigcup}\left\{\{(p,\{u\})\}: p \in E, u \in \Lambda_{F}(p)\right\}=\tilde{\bigcup}\{\{(p,\{u\})\}:(p,\{u\}) \tilde{\epsilon} F\} .
\end{aligned}
$$

Proposition 3.3 Let $F_{1}, F_{2} \in S(U)$. The following holds:

$$
F_{1} \tilde{\subseteq} F_{2} \quad \Leftrightarrow \quad \forall_{\alpha}\left(\alpha \tilde{\in} F_{1} \Rightarrow \alpha \tilde{\epsilon} F_{2}\right)
$$

Proof Let $F_{1}, F_{2} \in S(U)$. We have

$$
\begin{aligned}
& \forall_{p \in E} \Lambda_{F_{1}}(p) \subseteq \Lambda_{F_{2}}(p) \quad \Leftrightarrow \quad \forall_{p \in E} \forall_{u \in U}\left(u \in \Lambda_{F_{1}}(p) \Rightarrow u \in \Lambda_{F_{2}}(p)\right) \\
& \Leftrightarrow \quad \forall_{\alpha=(p,\{u\})}\left(\alpha \tilde{\in} F_{1} \Rightarrow \alpha \tilde{\in} F_{2}\right) .
\end{aligned}
$$

\section{Soft compact topological spaces}

In this section we introduce the definitions and the basic properties concerning soft compact topological spaces, which will be useful in the next sections.

The foundations of the theory of soft topological spaces were given by Çağman et al. in [4]. Let us recall the definition of a soft topological space.

Definition 4.1 A soft topology on $F \in S(U)$ is a collection $\tilde{\tau}$ of soft subsets of $F$ satisfying:

1. $\tilde{\varnothing}, F \in \tilde{\tau}$,

2. $\left\{F_{i}\right\}_{i \in I} \subseteq \tilde{\tau} \Rightarrow \tilde{\bigcup}_{i \in I} F_{i} \in \tilde{\tau}$,

3. $F_{1}, F_{2} \in \tilde{\tau} \Rightarrow F_{1} \tilde{\cap} F_{2} \in \tilde{\tau}$.

If $\tilde{\tau}$ is a soft topology on $F$, then the pair $(F, \tilde{\tau})$ is called a soft topological space.

The basic definitions and properties regarding soft topological spaces are also introduced in [4].

Using the introduced notion of soft element, the following proposition gives a natural characterization of soft open sets.

Proposition 4.1 Let $(F, \tilde{\tau})$ be a soft topological space. A soft set $V \tilde{\subseteq} F$ is soft open if and only iffor each $\alpha \tilde{\in} V$ there exists a soft set $W \in \tilde{\tau}$ such that $\alpha \tilde{\in} W \tilde{\subseteq} V$.

Proof Let $V \in \tilde{\tau}$. Then, clearly, for each $\alpha \tilde{\epsilon} V$ we have $\alpha \tilde{\epsilon} V \tilde{\subseteq} V$. 
Let $V \tilde{\subseteq} F$ be such that for each $\alpha \tilde{\epsilon} V$ there exists a soft open soft set $W(\alpha)$ such that $\alpha \in W(\alpha) \tilde{\subseteq} V$. Then, by Proposition 3.2 and Proposition 3.1(2), we obtain

$$
V=\tilde{\bigcup}\{\{\alpha\}: \alpha \tilde{\in} V\} \tilde{\subseteq} \tilde{\bigcup} W(\alpha) \tilde{\subseteq} V .
$$

Thus, $V=\tilde{U} W(\alpha) \in \tilde{\tau}$.

Definition 4.2 Let $(F, \tilde{\tau})$ be a soft topological space and let $G \tilde{\subseteq} F$. The soft topology on $G$ induced by the soft topology $\tilde{\tau}$ is the family $\tilde{\tau}_{G}$ of the soft subsets of $G$ of the form

$$
\tilde{\tau}_{G}=\{V \tilde{\cap} G: V \in \tilde{\tau}\}
$$

It is easy to verify that the family $\tilde{\tau}_{G}$ is a soft topology on $G$. The soft topological space $\left(G, \tilde{\tau}_{G}\right)$ is called a soft topological subspace of $(F, \tilde{\tau})$.

Definition 4.3 We say that a soft topological space $(F, \tilde{\tau})$ is soft Hausdorff if for each $\alpha, \beta \tilde{\epsilon} F, \alpha \neq \beta$ there exist $V_{1}, V_{2} \in \tilde{\tau}$ such that $V_{1} \tilde{\cap} V_{2}=\tilde{\varnothing}$ and $\alpha \tilde{\epsilon} V_{1}, \beta \tilde{\epsilon} V_{2}$.

Definition 4.4 Let $(F, \tilde{\tau})$ be a soft topological space and let $K \tilde{\subseteq} F$. The family $\left\{V_{i}\right\}_{i \in I} \subseteq \tilde{\tau}$ is called a soft open cover of $K$ if $K \tilde{\subseteq} \tilde{U}_{\alpha \in I} V_{i}$.

Definition 4.5 A soft topological space $(K, \tilde{\tau})$ is called soft compact if for each soft open cover $\left\{V_{i}\right\}_{i \in I}$ of $K$ there exist $i_{1}, i_{2}, \ldots, i_{k} \in I, k \in \mathbb{N}$ such that $K \tilde{\subseteq} \tilde{U}_{n=1}^{k} V_{i_{n}}$.

Definition 4.6 Let $(F, \tilde{\tau})$ be a soft topological space and let $K \tilde{\subseteq} F$. We say that the soft set $K$ is compact in $(F, \tilde{\tau})$ if the soft topological space $\left(K, \tilde{\tau}_{K}\right)$ is soft compact.

Proposition 4.2 Let $(F, \tilde{\tau})$ be a soft Hausdorff topological space. Then every soft compact set in $F$ is soft closed in $F$.

Proof Let $K$ be a soft compact set in $(F, \tilde{\tau})$ and let $\alpha \tilde{\in} K^{\tilde{c}}$. For every $\beta \tilde{\in} K$, let $U(x), V(x) \in \tilde{\tau}$ be such that $U(x) \tilde{\cap} V(x)=\tilde{\varnothing}$ and $\alpha \tilde{\in} U(x), \beta \tilde{\in} V(x)$. From the soft compactness of $K$, there exist $\beta_{1}, \ldots, \beta_{k} \tilde{\in} K$ such that $K \tilde{\subseteq} V\left(x_{1}\right) \tilde{\cup} \ldots \tilde{\cup} V\left(x_{k}\right)$. Denote $U=U\left(x_{1}\right) \tilde{\cap} \ldots \tilde{\cap} U\left(x_{k}\right)$ and $V=V\left(x_{1}\right) \tilde{\cap} \cdots \tilde{\cap} V\left(x_{k}\right)$. Then $\alpha \tilde{\epsilon} U \in \tilde{\tau}, U \tilde{\cap} V=\tilde{\varnothing}$ and thus $U \tilde{\cap} K=\tilde{\varnothing}$, which gives that $\alpha \tilde{\in} U \tilde{\subseteq} K^{\tilde{c}}$ and consequently, by Proposition $4.1, K$ is soft closed.

\section{Soft mappings}

In this section we establish a new concept of a soft mapping, formulate related definitions and prove the properties of soft mappings.

We start with the definition of the Cartesian product and soft relation, which were firstly defined by Babitha and Sunil in [3]. We may read them as follows.

Definition 5.1 Let $F_{1}, F_{2} \in S(U)$. The soft Cartesian product of $F_{1}$ and $F_{2}$, denoted by $F_{1} \tilde{\times} F_{2}$, is a soft set on $U \times U$ of the form

$$
F_{1} \tilde{\times} F_{2}=\left\{\left(\left(p_{1}, p_{2}\right), \Lambda_{F_{1}}\left(p_{1}\right) \times \Lambda_{F_{2}}\left(p_{2}\right)\right): p_{1}, p_{2} \in E\right\} .
$$


Example 5.1 Let $U=\left\{u_{1}, u_{2}, u_{3}\right\}, E=\left\{p_{1}, p_{2}, p_{3}\right\}$. Define $F_{1}, F_{2} \in S(U)$ as follows: $F_{1}=$ $\left\{\left(p_{1},\left\{u_{1}, u_{2}\right\}\right)\right\}, F_{2}=\left\{\left(p_{2},\left\{u_{1}\right\}\right),\left(p_{3},\left\{u_{1}, u_{3}\right\}\right)\right\}$. Then the soft product of $F_{1}, F_{2}$ is of the form

$$
\begin{aligned}
F_{1} \tilde{\times} F_{2}= & \left\{\left(\left(p_{1}, p_{1}\right),\left\{u_{1}, u_{2}\right\} \times \varnothing\right),\left(\left(p_{1}, p_{2}\right),\left\{u_{1}, u_{2}\right\} \times\left\{u_{1}\right\}\right),\left(\left(p_{1}, p_{3}\right),\left\{u_{1}, u_{2}\right\} \times\left\{u_{1}, u_{3}\right\}\right),\right. \\
& \left(\left(p_{2}, p_{1}\right), \varnothing \times \varnothing\right),\left(\left(p_{2}, p_{2}\right), \varnothing \times\left\{u_{1}\right\}\right),\left(\left(p_{2}, p_{3}\right), \varnothing \times\left\{u_{1}, u_{3}\right\}\right), \\
& \left.\left(\left(p_{3}, p_{1}\right), \varnothing \times \varnothing\right),\left(\left(p_{3}, p_{2}\right), \varnothing \times\left\{u_{1}\right\}\right),\left(\left(p_{3}, p_{3}\right), \varnothing \times\left\{u_{1}, u_{3}\right\}\right)\right\} \\
= & \left\{\left(\left(p_{1}, p_{2}\right),\left\{\left(u_{1}, u_{1}\right),\left(u_{2}, u_{1}\right)\right\}\right),\left(\left(p_{1}, p_{3}\right),\left\{\left(u_{1}, u_{1}\right),\left(u_{1}, u_{3}\right),\left(u_{2}, u_{1}\right),\left(u_{2}, u_{3}\right)\right\}\right)\right\} .
\end{aligned}
$$

Definition 5.2 Let $F_{1}, F_{2} \in S(U)$. A soft set $R \in S(U)$ is called a soft relation from $F_{1}$ to $F_{2}$ if $R \tilde{\subseteq} F_{1} \tilde{\times} F_{2}$, i.e., $R$ is a soft set of the form

$$
R=\left\{\left((p, q), U_{p} \times U_{q}\right): p, q \in E, U_{p} \subseteq \Lambda_{F_{1}}(p), U_{q} \subseteq \Lambda_{F_{2}}(q)\right\} .
$$

If $\left((p, q), U_{p} \times U_{q}\right) \in R$, then we will write $\left(p, U_{p}\right) R\left(q, U_{q}\right)$.

Example 5.2 Let $F_{1}, F_{2}$ be as in Example 5.1. Then

$$
R=\left\{\left(\left(p_{1}, p_{2}\right),\left\{\left(u_{1}, u_{1}\right)\right\}\right),\left(\left(p_{1}, p_{3}\right),\left\{\left(u_{1}, u_{3}\right),\left(u_{2}, u_{3}\right)\right\}\right)\right\}
$$

is an example of soft relation from $F_{1}$ to $F_{2}$. Consequently, we can write

$$
\left(p_{1},\left\{u_{1}\right\}\right) R\left(p_{2},\left\{u_{1}\right\}\right), \quad\left(p_{1},\left\{u_{1}, u_{2}\right\}\right) R\left(p_{3},\left\{u_{3}\right\}\right) .
$$

Now, we will introduce a new definition of a soft mapping.

Definition 5.3 Let $F, G \in S(U)$. A soft relation $T \tilde{\subseteq} F \tilde{\times} G$ is called a soft mapping from $F$

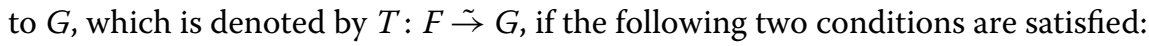

(SM1) for each soft element $\alpha \tilde{\epsilon} F$, there exists only one soft element $\beta \tilde{\epsilon} G$ such that $\alpha T \beta$ (which will be noted as $T(\alpha)=\beta$ );

(SM2) for each empty soft element $\alpha \tilde{\in} F, T(\alpha)$ is an empty soft element of $G$.

Remark 5.1 Observe that the above established definition of soft mapping is different from the notion of soft function introduced by Babitha and Sunil in [3] and also differs from the concept of soft mapping by Kharal and Ahmad in [7] and by Majumdar and Samanta in [6]. Example 5.3 of a soft mapping is not a soft mapping in the sense introduced by the mentioned authors. This new approach, strictly related with the introduced notion of soft element, enables us to obtain a natural (similar to classical mappings) behavior of soft mappings. Please compare the rest of this section. Moreover, this type of soft mappings will play a fundamental role in obtaining a fixed-point theorem in the last section.

Example 5.3 Let $F_{1}, F_{2} \in S(U)$ be as in Example 5.1 and let $T \tilde{\subseteq} F_{1} \tilde{\times} F_{2}$ be of the form

$$
\begin{aligned}
T= & \left(\left(p_{1}, p_{1}\right), \varnothing \times \varnothing\right),\left(\left(p_{1}, p_{2}\right),\left\{u_{1}\right\} \times\left\{u_{1}\right\}\right),\left(\left(p_{1}, p_{3}\right),\left\{u_{2}\right\} \times\left\{u_{3}\right\}\right), \\
& \left.\left(\left(p_{2}, p_{1}\right), \varnothing \times \varnothing\right),\left(\left(p_{3}, p_{1}\right), \varnothing \times \varnothing\right)\right\} .
\end{aligned}
$$


Then $T$ is a soft mapping from $F$ to $G$ and can be written in a more intuitive way:

$$
\begin{aligned}
& T\left(p_{1}, \varnothing\right)=\left(p_{1}, \varnothing\right), \quad T\left(p_{2}, \varnothing\right)=\left(p_{1}, \varnothing\right), \\
& T\left(p_{1},\left\{u_{1}\right\}\right)=\left(p_{2},\left\{u_{1}\right\}\right), \quad T\left(p_{3}, \varnothing\right)=\left(p_{1}, \varnothing\right), \\
& T\left(p_{1},\left\{u_{2}\right\}\right)=\left(p_{3},\left\{u_{3}\right\}\right) .
\end{aligned}
$$

Definition 5.4 Let $F, G \in S(U)$ and let $T: F \stackrel{\sim}{\rightarrow} G$ be a soft mapping. The image of $X \tilde{\simeq} F$ under soft mapping $T$ is the soft set, denoted by $T(X)$, of the form

$$
T(X)=\bigcup_{\alpha \tilde{\in} X}^{\sim}\{T(\alpha)\} .
$$

It is clear that $T(\tilde{\varnothing})=\tilde{\varnothing}$ for each soft mapping $T$.

Definition 5.5 Let $F, G \in S(U)$ and let $T: F \stackrel{\sim}{\rightarrow} G$ be a soft mapping. The inverse of $Y \subseteq G$ under soft mapping $T$ is the soft set, denoted by $T^{-1}(Y)$, of the form

$$
T^{-1}(Y)=\tilde{\bigcup}\{\{\alpha\}: \alpha \tilde{\epsilon} F, T(\alpha) \tilde{\epsilon} Y\} .
$$

Remark 5.2 Let us observe that the condition (SM2) of Definition 5.3 implies that the inverse under soft mapping $T$ in Definition 5.5 is well defined. In particular, the inverse of the empty soft set under $T$ is always a soft subset of $F$.

Example 5.4 Let $U=E=\mathbb{N}$ and let $F, G \in S(U)$ be defined as follows:

$$
F=\{(p,\{p, p+1\}): p \in E\}, \quad G=\{(p,\{2 p\}): p \in E\} .
$$

Define a soft mapping $T: F \stackrel{\sim}{\rightarrow} G$ by the formula

$$
\begin{aligned}
& T(p,\{p\})=(2 p,\{4 p\}), \quad T(p,\{p+1\})=(4 p+4,\{8 p+8\}), \\
& T(p, \varnothing)=(p, \varnothing), \quad p \in E .
\end{aligned}
$$

Taking $X=F$, we have

$$
\begin{aligned}
T(X) & =\tilde{\bigcup}\{T(p, \varnothing)\} \tilde{\cup} \bigcup_{p \in E}^{\tilde{U}}\{T(p,\{p\})\} \tilde{\cup} \bigcup_{p \in E}^{\tilde{U}}\{T(p,\{p+1\})\} \\
& =\tilde{\bigcup_{p \in E}}\{(p, \varnothing)\} \tilde{\cup} \tilde{\bigcup}\{(2 p,\{4 p\})\} \tilde{\cup} \bigcup_{p \in E}\{(4 p+4,\{8 p+8\})\} \\
& =\{(2 p,\{4 p\}): p \in E\} .
\end{aligned}
$$

Now, we present the basic properties of soft mappings. The proofs are simple and hence omitted.

Proposition 5.1 Let $F, G \in S(U), X, X_{1}, X_{2} \tilde{\subseteq} F, Y, Y_{1}, Y_{2} \subseteq$ $G$ and let $T: F \stackrel{\sim}{\rightarrow} G$ be a soft mapping. Then the following hold: 
1. $X_{1} \tilde{\simeq} X_{2} \Rightarrow T\left(X_{1}\right) \tilde{\subseteq} T\left(X_{2}\right)$

2. $Y_{1} \tilde{\subseteq} Y_{2} \Rightarrow T^{-1}\left(Y_{1}\right) \tilde{\subseteq} T^{-1}\left(Y_{2}\right)$,

3. $X \subseteq T^{-1}(T(X))$,

4. $T\left(T^{-1}(Y)\right) \tilde{\subseteq} Y$,

5. $T\left(X_{1} \tilde{\cup} X_{2}\right)=T\left(X_{1}\right) \tilde{\cup} T\left(X_{2}\right)$,

6. $T\left(X_{1} \tilde{\cap} X_{2}\right) \tilde{\subseteq} T\left(X_{1}\right) \tilde{\cap} T\left(X_{2}\right)$,

7. $T^{-1}\left(Y_{1} \tilde{\cup} Y_{2}\right)=T^{-1}\left(Y_{1}\right) \tilde{\cup} T^{-1}\left(Y_{2}\right)$,

8. $T^{-1}\left(Y_{1} \tilde{\cap} Y_{2}\right)=T^{-1}\left(Y_{1}\right) \tilde{\cap} T^{-1}\left(Y_{2}\right)$.

The formulae (5)-(8) are also true for the generalized soft operations $\tilde{\cup}$ and $\tilde{\cap}$.

Definition 5.6 Let $(F, \tilde{\tau}),(G, \tilde{v})$ be soft topological spaces and let $T: F \stackrel{\rightarrow}{\rightarrow} G$ be a soft mapping. We say that $T$ is a soft continuous mapping (with respect to the soft topologies $\tilde{\tau}$ and $\tilde{v}$ ) if for each $V \in \tilde{v}, T^{-1}(V) \in \tilde{\tau}$ (i.e., the inverse of a soft open set is a soft open set).

Example 5.5 Let $U=\left\{u_{1}, u_{2}, u_{3}\right\}, E=\left\{p_{1}, p_{2}, p_{3}\right\}$ and let $F \in S(U)$ be of the form $F=$ $\left\{\left(p_{1},\left\{u_{1}, u_{2}\right\}\right),\left(p_{2},\left\{u_{2}, u_{3}\right\}\right)\right\}$. Consider the family $\tilde{\tau}$ of soft subsets of $F$

$$
\tilde{\tau}=\left\{\tilde{\varnothing}, F,\left\{\left(p_{1},\left\{u_{2}\right\}\right)\right\},\left\{\left(p_{1},\left\{u_{2}\right\}\right),\left(p_{2},\left\{u_{3}\right\}\right)\right\},\left\{\left(p_{1},\left\{u_{1}, u_{2}\right\}\right),\left(p_{2},\left\{u_{2}\right\}\right)\right\}\right\} .
$$

Then $(F, \tilde{\tau})$ is a soft topological space (see Example 3 in [4]).

Now, taking a soft mapping $T_{1}: F \stackrel{\sim}{\rightarrow} F$ as follows:

$$
\begin{array}{ll}
T_{1}\left(p_{1}, \varnothing\right)=\left(p_{2}, \varnothing\right), & T_{1}\left(p_{1},\left\{u_{1}\right\}\right)=\left(p_{2},\left\{u_{3}\right\}\right), \\
T_{1}\left(p_{1},\left\{u_{2}\right\}\right)=\left(p_{1},\left\{u_{1}\right\}\right), & T_{1}\left(p_{2}, \varnothing\right)=\left(p_{1}, \varnothing\right), \\
T_{1}\left(p_{2},\left\{u_{2}\right\}\right)=\left(p_{2},\left\{u_{3}\right\}\right), & T_{1}\left(p_{2},\left\{u_{3}\right\}\right)=\left(p_{1},\left\{u_{2}\right\}\right), \\
T_{1}\left(p_{3}, \varnothing\right)=\left(p_{3}, \varnothing\right), &
\end{array}
$$

we obtain that for $V=\left\{\left(p_{1},\left\{u_{2}\right\}\right)\right\} \in \tilde{\tau}, T_{1}^{-1}(V)=\left\{\left(p_{2},\left\{u_{3}\right\}\right)\right\} \notin \tilde{\tau}$ and thus $T_{1}$ is not a soft continuous mapping.

If we consider a soft mapping $T_{2}: F \stackrel{\sim}{\rightarrow} F$ of the form

$$
\begin{array}{ll}
T_{2}\left(p_{1}, \varnothing\right)=\left(p_{2}, \varnothing\right), & T_{2}\left(p_{2},\left\{u_{2}\right\}\right)=\left(p_{1},\left\{u_{2}\right\}\right), \\
T_{2}\left(p_{1},\left\{u_{1}\right\}\right)=\left(p_{1},\left\{u_{2}\right\}\right), & T_{2}\left(p_{2},\left\{u_{3}\right\}\right)=\left(p_{2},\left\{u_{2}\right\}\right), \\
T_{2}\left(p_{1},\left\{u_{2}\right\}\right)=\left(p_{1},\left\{u_{2}\right\}\right), & T_{2}\left(p_{3}, \varnothing\right)=\left(p_{3}, \varnothing\right), \\
T_{2}\left(p_{2}, \varnothing\right)=\left(p_{1}, \varnothing\right), &
\end{array}
$$

then it is easy to verify that $T_{2}^{-1}(V) \in \tilde{\tau}$ for each $V \in \tilde{\tau}$ and hence $T_{2}$ is a soft continuous mapping.

Proposition 5.2 Let $(K, \tilde{\tau})$ be a soft compact topological space and let $T: K \stackrel{\rightarrow}{\rightarrow} K$ be a soft continuous mapping. Then $T(K)$ is a soft compact set in $(K, \tilde{\tau})$. 
Proof Let $\left\{V_{i}\right\}_{i \in I} \subseteq \tilde{\tau}$ be such that $T(K) \tilde{\subseteq} \tilde{U}_{i \in I} V_{i}$. Due to the soft continuity of $T$, we get that $\left\{T^{-1}\left(V_{i}\right)\right\}_{i \in I}$ is a family of soft open sets. Moreover, we have

$$
K \tilde{\subseteq} T^{-1}(T(K)) \tilde{\subseteq} T^{-1}\left(\tilde{\bigcup}_{i \in I}^{\tilde{V}} V_{i}\right)=\bigcup_{i \in I}^{\tilde{T}} T^{-1}\left(V_{i}\right) .
$$

From the soft compactness of $K$, there exist $i_{1}, i_{2}, \ldots, i_{k} \in I, k \in \mathbb{N}$ such that

$$
K \tilde{\subseteq} T^{-1}\left(V_{i_{1}}\right) \tilde{\cup} T^{-1}\left(V_{i_{2}}\right) \tilde{\cup} \cdots \tilde{\cup} T^{-1}\left(V_{i_{k}}\right) .
$$

By the above we obtain

$$
T(K) \tilde{\subseteq} T\left(T^{-1}\left(V_{i_{1}}\right) \tilde{\cup} T^{-1}\left(V_{i_{2}}\right) \tilde{\cup} \cdots \tilde{\cup} T^{-1}\left(V_{i_{k}}\right)\right) \tilde{\subseteq} V_{i_{1}} \tilde{\cup} V_{i_{2}} \tilde{\cup} \ldots \tilde{\cup} V_{i_{k}} .
$$

Thus, $T(K)$ is a soft compact set.

\section{Fixed points of soft mappings - the main result}

We start this section from the definition of a fixed point of a soft mapping.

Definition 6.1 Let $F \in S(U)$ be a soft set and let $T: F \tilde{\rightarrow} F$ be a soft mapping. A soft element $\alpha \tilde{\in} F$ is called a fixed point of $T$ if $T(\alpha)=\alpha$.

Example 6.1 Let $T_{2}$ be a soft mapping defined in Example 5.5. Then the soft elements $\left(p_{1},\left\{u_{2}\right\}\right),\left(p_{3}, \varnothing\right)$ are the fixed points of $T_{2}$.

Proposition 6.1 Let $(F, \tilde{\tau})$ be a soft compact topological space and let $\left\{C_{n}: n \in \mathbb{N}\right\}$ be a family of soft subsets of $F$ satisfying:

1. $C_{n} \neq \tilde{\varnothing}$ for each $n \in \mathbb{N}$,

2. $C_{n}$ is soft closed for each $n \in \mathbb{N}$,

3. $C_{n+1} \tilde{\subseteq} C_{n}$ for each $n \in \mathbb{N}$.

Then $\tilde{\bigcap}_{n \in \mathbb{N}} C_{n} \neq \tilde{\varnothing}$.

Proof Suppose that $\tilde{\bigcap}_{n \in \mathbb{N}} C_{n}=\tilde{\varnothing}$. Let us observe that for each $n \in \mathbb{N}$, from (2), $C_{n}^{\tilde{c}}$ is a soft open set and

$$
F \tilde{\subseteq} I_{\tilde{E}}=\tilde{\varnothing}^{\tilde{c}}=\left(\bigcap_{n \in \mathbb{N}} C_{n}\right)^{\tilde{c}}=\bigcup_{n \in \mathbb{N}} C_{n}^{\tilde{c}} .
$$

Due to the soft compactness of $F$, there exist $i_{1}, i_{2}, \ldots, i_{k} \in \mathbb{N}, 0<i_{1}<i_{2}<\cdots<i_{k}, k \in \mathbb{N}$ such that

$$
F \tilde{\subseteq} C_{i_{1}}^{\tilde{c}} \tilde{\cup} C_{i_{2}}^{\tilde{c}} \tilde{\cup} \cdots \tilde{\cup} C_{i_{k}}^{\tilde{c}}
$$

By the above and by (3), we have

$$
C_{i_{k}} \tilde{\subseteq} F \tilde{\subseteq}\left(C_{i_{1}} \tilde{\cap} C_{i_{2}} \tilde{\cap} \cdots \tilde{\cap} C_{i_{k}}\right)^{\tilde{c}}=C_{i_{k}}^{\tilde{c}}=I_{\tilde{E}} \tilde{\backslash} C_{i_{k}},
$$

which, due to (1), is impossible. 
Proposition 6.2 Let $(F, \tilde{\tau})$ be a soft topological space and let $T: F \stackrel{\sim}{\rightarrow} F$ be a soft mapping such that for each nonempty soft element $\alpha \tilde{\in} F, T(\alpha)$ is a nonempty soft element of $F$. If $\tilde{\bigcap}_{n \in \mathbb{N}} T^{n}(F)$ contains only one nonempty soft element $\alpha \in F$, then $\alpha$ is a unique fixed point of $T$.

Proof Observe that $T^{n}(F) \tilde{\subseteq} T^{n-1}(F)$ for each $n \in \mathbb{N}$. Let $\alpha$ be a nonempty soft element of $F$ such that $\alpha \tilde{\in} \tilde{\bigcap}_{n \in \mathbb{N}} T^{n}(F)$. Then we get $\{\alpha\} \tilde{\subseteq} \tilde{\bigcap}_{n \in \mathbb{N}} T^{n}(F)$ and consequently

$$
T(\{\alpha\}) \tilde{\subseteq} T\left(\bigcap_{n \in \mathbb{N}} T^{n}(F)\right) \tilde{\subseteq} \bigcap_{n \in \mathbb{N}} T^{n+1}(F) \tilde{\subseteq} \bigcap_{n \in \mathbb{N}} T^{n}(F)=\{\alpha\} .
$$

Since $T(\alpha)$ is a nonempty soft element, we obtain that $T(\alpha)=\alpha$.

Remark 6.1 The assertion about the nonempty soft values in Proposition 6.2 is necessary.

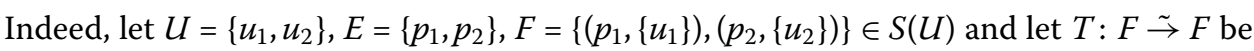
of the form

$$
\begin{aligned}
& T\left(p_{1}, \varnothing\right)=\left(p_{2}, \varnothing\right), \quad T\left(p_{2}, \varnothing\right)=\left(p_{2},\left\{u_{2}\right\}\right), \\
& T\left(p_{1},\left\{u_{1}\right\}\right)=\left(p_{2},\left\{u_{2}\right\}\right), \quad T\left(p_{2},\left\{u_{2}\right\}\right)=\left(p_{2}, \varnothing\right) .
\end{aligned}
$$

Then $\tilde{\bigcap}_{n \in \mathbb{N}} T^{n}(F)=\left\{\left(p_{2},\left\{u_{2}\right\}\right)\right\}$, but $T$ is a fixed point-free soft mapping.

Theorem 6.1 Let $(K, \tilde{\tau})$ be a soft compact Hausdorff topological space and let $T: K \stackrel{\sim}{\rightarrow} K$ be a soft continuous mapping such that:

1. for each nonempty soft element $\alpha \tilde{\in} K, T(\alpha)$ is a nonempty soft element of $K$,

2. for each soft closed set $X \tilde{\subseteq} K$, if $T(X)=X$, then $X$ contains only one nonempty soft element of $K$.

Then there exists a unique nonempty soft element $\alpha \tilde{\in} K$ such that $T(\alpha)=\alpha$.

Proof Consider a family of soft subsets of $K$ of the form $C_{1}=T(K), C_{2}=T\left(C_{1}\right)=T^{2}(K)$, $\ldots, C_{n}=T\left(C_{n-1}\right)=T^{n}(K), n \in \mathbb{N}, \ldots$ It is clear that $C_{n} \tilde{\subseteq} C_{n-1}$ for each $n \in \mathbb{N}$. By Proposition 4.2, for each $n \in \mathbb{N}, C_{n}$ is soft closed and, due to Proposition 6.1, a soft set $D$ of the form $D=\tilde{\bigcap}_{n \in \mathbb{N}} C_{n}$ is nonempty.

Let us observe that

$$
T(D)=T\left(\bigcap_{n \in \mathbb{N}} T^{n}(K)\right) \tilde{\subseteq} \bigcap_{n \in \mathbb{N}} T^{n+1}(K) \tilde{\subseteq} \bigcap_{n \in \mathbb{N}} T^{n}(K)=D .
$$

In order to show that $D \tilde{\subseteq} T(D)$, suppose that there exists $\alpha \tilde{\in} D$ such that $\alpha \tilde{\notin} T(D)$. Denote $E_{n}=T^{-1}(\{\alpha\}) \tilde{\cap} C_{n}$. Let us observe that $E_{n} \neq \tilde{\varnothing}$ and $E_{n} \tilde{\subseteq} E_{n-1}$ for each $n \in \mathbb{N}$. By Proposition 6.1, there exists a nonempty soft element $\beta \tilde{\epsilon} T^{-1}(\{\alpha\}) \tilde{\cap} D$ and thus $\alpha=T(\beta) \tilde{\epsilon} T(D)$, which is a contradiction. In consequence, $T(D)=D$ that, by (2) and Proposition 6.2, completes the proof.

The assumption about the soft compactness in Theorem 6.1 is not superfluous. To see this, consider the following example. 
Example 6.2 Let $U=\mathbb{R}, E=\mathbb{Z}$ and let $F \in S(U)$ be a soft set of the form

$$
F=\{(p,(p, p+1]): p \in E\} .
$$

When we consider a soft topology $\tilde{\tau}$ as a family of all soft subsets of $F$, then clearly $(F, \tilde{\tau})$ is not a soft compact topological space. Now, take a soft function $T: F \stackrel{\sim}{\rightarrow} F$ by the formulae:

$$
\begin{aligned}
& T(p, \varnothing)=(p+1, \varnothing), \quad p \in E, \\
& T(p,\{u\})=(p+1,\{u+1\}), \quad p \in E, u \in(p, p+1] .
\end{aligned}
$$

Then $T$ is obviously a soft continuous mapping, $T(X) \neq X$ for any $X \tilde{\subseteq} F$ and, clearly, $T$ does not have a fixed point.

\section{Competing interests}

The author declares that he has no competing interests.

Received: 15 October 2012 Accepted: 26 June 2013 Published: 11 July 2013

\section{References}

1. Molodtsov, DA: Soft set theory - first results. Comput. Math. Appl. 62, 351-358 (2011)

2. Ali, MI, Feng, F, Liu, XY, Min, WK, Shabir, M: On some new operations in soft set theory. Comput. Math. Appl. 62, 351-358 (2011)

3. Babitha, KV, Suntil, JJ: Soft set relations and functions. Comput. Math. Appl. 60, 1840-1849 (2010)

4. Çağman, N, Karataş, S, Enginoglu, S: Soft topology. Comput. Math. Appl. 62, 351-358 (2011)

5. Maji, PK, Biswas, R, Roy, AR: Soft set theory. Comput. Math. Appl. 45, 555-562 (2003)

6. Majumdar, P, Samanta, SK: On soft mappings. Comput. Math. Appl. 60, 2666-2672 (2010)

7. Kharal, A, Ahmad, B: Mappings on fuzzy soft classes. Adv. Fuzzy Syst. (2009). doi:10.1155/2009/407890

8. Meng, D, Zhang, X, Qin, K: Soft rough fuzzy sets and soft fuzzy rough sets. Comput. Math. Appl. 62, 4635-4645 (2011)

9. Tanay, B, Kandemir, MB: Topological structure of fuzzy soft sets. Comput. Math. Appl. 61, 2952-2957 (2011)

10. Tarafdar, E, Yuan, XZ: Set-valued topological contractions. Appl. Math. Lett. 6, 79-81 (1995)

\section{Submit your manuscript to a SpringerOpen ${ }^{\circ}$ journal and benefit from:}

- Convenient online submission

Rigorous peer review

- Immediate publication on acceptance

- Open access: articles freely available online

- High visibility within the field

- Retaining the copyright to your article 